\title{
Expression of angiogenic regulators and skeletal muscle capillarity in selectively bred high aerobic capacity mice
}

\author{
Gerald N. Audet ${ }^{1}$, Thomas H. Meek ${ }^{2}$, Theodore Garland $\mathrm{Jr}^{2}$ and I. Mark Olfert ${ }^{1}$ \\ ${ }^{1}$ West Virginia University School of Medicine, Center for Cardiovascular and Respiratory Sciences, Division of Exercise Physiology, \\ Morgantown, WV 26508, USA \\ ${ }^{2}$ University of California-Riverside, Department of Biology, Riverside, CA 92521, USA
}

Selective breeding for high voluntary wheel running in untrained mice has resulted in a 'mini muscle' (MM) phenotype, which has increased skeletal muscle capillarity compared with muscles from non-selected control lines. Vascular endothelial growth factor (VEGF) and thrombospondin-1 (TSP-1) are essential mediators of skeletal muscle angiogenesis; thus, we hypothesized that untrained MM mice with elevated muscle capillarity would have higher basal VEGF expression and lower basal TSP-1 expression, and potentially an exaggerated VEGF response to acute exercise. We examined skeletal muscle morphology and skeletal muscle protein expression of VEGF and TSP-1 in male mice from two (untrained) mouse lines selectively bred for high exercise capacity (MM and Non-MM), as well as one non-selected control mouse line (normal aerobic capacity). In the MM mice, gastrocnemius (GA) and plantaris (PLT) muscle capillarity (i.e. capillary-to-fibre ratio and capillary density) were greater compared with control mice $(P<0.05)$. In Non-MM mice, only muscle capillarity in PLT was greater than in control mice $(P<0.001)$. The soleus $(\mathrm{SOL})$ showed no statistical differences in muscle capillarity among groups. In the GA, MM mice had $58 \%$ greater basal VEGF $(P<0.05)$, with no statistical difference in basal TSP-1 when compared with control mice. In the PLT, MM mice had a 79\% increase in basal VEGF $(P<0.05)$ and a 39\% lower basal TSP-1 $(P<0.05)$ compared with the control animals. Non-MM mice showed no difference in basal VEGF in either the GA or the PLT compared with control mice. In contrast, basal TSP-1 was elevated in the PLT, but not in the GA, of Non-MM mice compared with control mice. Neither VEGF nor TSP-1 was significantly different in SOL muscle among the three mouse lines. In response to acute exercise, MM mice displayed a 41 and $28 \%$ increase $(P<0.05)$ in VEGF in the GA and PLT, respectively, whereas neither control nor Non-MM mice showed a significant VEGF response to acute exercise. In contrast, TSP-1 levels were decreased by $90 \%$ in GA $(P<0.05)$ but increased by $50 \%$ in PLT $(P<0.05)$ in response to acute exercise in MM mice. The SOL showed no response to exercise for either VEGF or TSP-1 for any of the mouse lines. These data, with the exception of the Non-MM plantaris muscle, suggest that elevated capillarity is associated with altered balance between positive and negative angiogenic regulators (i.e. VEGF versus TSP-1, respectively). Based on the greater capillarity and significant VEGF response to exercise in MM mice, these data suggest that VEGF expression may, at least in part, be genetically determined.

(Received 11 February 2011; accepted after revision 25 July 2011; first published online 31 July 2011)

Corresponding author I. M. Olfert: West Virginia University School of Medicine, Center for Cardiovascular and Respiratory Sciences, Division of Exercise Physiology, PO Box 9105, Morgantown, WV 26508, USA.

Email: molfert@hsc.wvu.edu 
The importance of genetic influences in determining aerobic exercise capacity has been highlighted by selective breeding approaches used to developed high aerobic capacity lines of rodents (Swallow et al. 2009; Feder et al. 2010). For example, Koch \& Britton (2001) have developed separate high (HCR) and low aerobic capacity rat (LCR) lines via respective breeding of rats for either high or low endurance capacity on a motorized treadmill. This has resulted in a diverging phenotype, where HCRs have a higher cardiovascular (Koch et al. 1999; Howlett et al. 2009) and respiratory capacity (Henderson et al. 2002) and increased skeletal muscle metabolism (Howlett et al. 2003), underpinning their greater innate aerobic endurance capacity compared with LCRs. Data obtained from this artificial selection experiment also highlights the importance of reduced/impaired physical activity for the pathogenesis of chronic disease, as the incidence of some cardiovascular risk factors is significantly greater in LCRs compared with HCRs (Wisloff et al. 2005).

Continuous selective breeding for high voluntary wheel running in laboratory house mice has also resulted in the development of four replicate lines of mice with a significantly increased aerobic exercise capacity (Swallow et al. 1998a,b; Garland et al. 2011). The 'gene pool' of the original outbred population of mice from which this experiment originated included a Mendelian recessive allele whose most notable feature is to cause a $50 \%$ reduction in hindlimb muscle mass when present in the homozygous condition (Garland et al. 2002; Houle-Leroy et al. 2003). This 'mini-muscle' phenotype was favoured by the selective breeding regimen and increased in frequency in two of the four replicate selected lines (Garland et al. 2002). Further study of the mini-muscle mice (MM mice) has indicated several distinct physiological characteristics that may be advantageous with respect to wheel running. The most distinct feature is a significant reduction in the mass of most hindlimb muscles, but also includes fibre-type alterations favouring more oxidative fibres, increased tibia length (Syme et al. 2005; Guderley et al. 2008; McGillivray et al. 2009; Wong et al. 2009), increased mitochondrial enzymatic activity (Guderley et al. 2006), a smaller overall body mass (Swallow et al. $1999)$ and an increased maximal oxygen uptake $\left(\dot{V}_{\mathrm{O}_{2} \max }\right)$ when tested in hypoxia (Rezende et al. 2006a,b). More recently, it has also been shown that MM mice exhibit greater capillarity in the gastrocnemius compared with mice that have normal-sized muscles, especially around the medium and large fibres $\left(>2000 \mu \mathrm{m}^{2}\right.$; Wong et al. 2009).

Given that oxygen delivery to skeletal muscle is dependent on gas exchange across the microvascular capillary bed, one important mechanism to increase oxygen delivery to working muscle is to increase the number and density of capillaries surrounding the fibres (Andersen \& Henriksson, 1977; Wagner, 2003). Two potentially important mediators in the angiogenic process are vascular endothelial growth factor (VEGF; a positive angiogenic factor) and thrombospondin-1 (TSP-1; a negative angiogenic factor). In normal skeletal muscle, it has been shown that acute aerobic exercise increases VEGF (Breen et al. 1996; Gavin \& Wagner, 2001; Olfert et al. 2001; Gustafsson et al. 2002; Lloyd et al. 2003) and TSP-1 gene expression (Olfert et al. 2006), and that chronic or repeated exercise bouts ultimately decrease TSP-1 mRNA while VEGF mRNA remains elevated (Gavin \& Wagner, 2001; Olfert et al. 2001; Waters et al. 2004). Indeed, the importance of VEGF in regulating skeletal muscle capillarity is emphasized by recent evidence demonstrating that significant losses in VEGF result in reduced skeletal muscle capillarity (Tang et al. 2004; Olfert et al. 2009) and altered vascular function (Lee et al. 2007). Moreover, training-induced skeletal muscle angiogenesis is absent in VEGF-deficient mice (Olfert et al. 2010). By the same token, the importance of TSP-1 expression in preventing hypercapillarization of the skeletal muscle vascular bed has been shown using TSP-1 knockout mice (Malek \& Olfert, 2009).

Given the importance of VEGF and TSP-1 to skeletal muscle structure and function, we sought to examine VEGF and TSP-1 expression from two of the four selected mouse lines with inherited high aerobic capacity [one with the MM phenotype and another without the MM phenotype (i.e. Non-MM)] compared with a non-selected control line. We chose to examine two high capacity lines in our study, MM (Garland designated line 3) and Non-MM (Garland designated line 8), in order to more generally evaluate the high aerobic capacity phenotype, and not only the MM phenotype, compared with a non-selected control line (Garland designated line 2). In essence, the Non-MM mouse serves as an additional high capacity experimental group compared with the control line, but also as an additional high capacity group compared with MM mice (which have a very distinctive mini-muscle phenotype). For each of the three mouse lines, we examined basal expression and the response to acute exercise of VEGF and TSP-1 protein in the triceps surae hindlimb muscle. As both the MM and the NonMM mice have increased maximal oxygen consumption and treadmill endurance capacity during forced exercise compared with mice from control lines (Meek et al. 2009), and it has been shown that MM mice display elevated gastrocnemius muscle capillarity (Wong et al. 2009), it was first hypothesized that Non-MM mice would also have elevated skeletal muscle capillarity compared with control mice and second, that basal levels of VEGF would be higher in both MM and Non-MM high aerobic capacity mice compared with control mice. Moreover, given that all three mouse lines were untrained, we reasoned that we might see an exaggerated gene response to acute exercise in the high aerobic capacity lines compared with 
control animals due to the selection of genetic influences (resulting from the selected breeding strategy) that would favour greater skeletal muscle capillarity and high aerobic capacity mice, in the absence of training. On the other hand, as the high aerobic capacity lines are expected to have elevated muscle capillarity (and in normal mice elevated skeletal muscle capillarity has been associated with reduced VEGF responsiveness to exercise, at least in the context of training), it could also be hypothesized that simply having elevated capillarity (even in the absence of training) might diminish or attenuate the VEGF response to exercise in the high capacity selected lines compared with control mice.

In this study, we report data from the triceps surae muscles (which are known to have varying oxidative and glycolytic capacity) in order to identify potential differences in the skeletal muscle angiogenic response between muscles; but more specifically, we sought to exploit the innate difference between the respective mouse lines as an approach that might be a useful in unravelling the environmental versus genetic influences on skeletal muscle vascularization.

\section{Methods}

\section{Animals}

Male MM mice (laboratory designation selected line 3, $n=12$ ), Non-MM mice (selected line $8, n=12$ ) and control mice (line 2, $n=12$ ) were bred and raised at the University of California, Riverside. For a comprehensive review on the breeding and selection process, please see Swallow et al. (1998a). Briefly, eight independent lines of mice were created in the early 1990s from a common population of outbred Hsd:ICR laboratory mice. All lines were provided with running wheels for 6 days when the mice were $\sim 6-8$ weeks of age. In the four selected lines, the top-running male and female from within each of $\sim 10$ families, each generation, were used for breeding and the continuation of the line. The breeders were selected for the greatest distance run on the fifth and sixth day of wheel exposure. The other four lines were bred at random with respect to the amount of running, and served as control lines. After $\sim 16$ generations, the four 'high runner' lines ran approximately 2.7 times as far as the control lines, and had begun to display a number of characteristics unique to these lines (Swallow et al. 1998b, 2001, 2009; Koteja et al. 1999). In the present study, the mouse lines we examined came from the 54th generation, at which time the four selected lines were running more than three times further than control lines on a daily basis (T. Garland, unpublished observations). All procedures that involved animals were approved by the University of California, Riverside, Institutional Animal Care and Use Committee.

\section{Acute exercise protocol}

A subgroup of mice $(n=6)$ in each line were randomly assigned to perform a single bout of acute exercise in order to access growth factor response to an angiogenic stimulus. These mice ran in a custom-built treadmill (Meek et al. 2009). The clear Plexiglass lane in which the mouse ran had dimensions $6.5 \mathrm{~cm}$ wide, $12.5 \mathrm{~cm}$ high and $44 \mathrm{~cm}$ long. All animals were familiarized with the treadmill for $5 \mathrm{~min}$ prior to the acute exercise bouts. After this initial period, mice ran for $1 \mathrm{~h}$ at $20 \mathrm{~m} \mathrm{~min}^{-1}$ with $10 \mathrm{deg}$ incline. A run time of $1 \mathrm{~h}$ was selected because it has been shown to elicit a robust exercise-induced angiogenic gene response in rodents (Breen et al. 1996; Olfert et al. 2001). Animals were killed $4 \mathrm{~h}$ after the acute exercise at a time point known to correspond to peak VEGF protein expression (I. M. Olfert, unpublished data).

\section{Tissue preparations}

Each animal was killed by $\mathrm{CO}_{2}$ inhalation, and the triceps surae [gastrocnemius (GA), soleus (SOL) and plantaris (PLT)] muscles from each leg were immediately excised. Muscles from the right leg were weighed and immediately flash frozen in liquid $\mathrm{N}_{2}$, then later processed for VEGF protein expression. Muscles from the left leg were mounted on cork disks and quickly frozen in isopentane cooled in liquid $\mathrm{N}_{2}$, then processed later for morphometrical analysis. All samples were then stored at $-80^{\circ} \mathrm{C}$ until processed for molecular or morphological analyses.

\section{Morphometry}

Frozen tissue was cut using a $-20^{\circ} \mathrm{C}$ cyromicrotome (Jung-Reichert Cryocut 1800; Cambridge Instruments, Nussloch, Germany) to yield $10-\mu \mathrm{m}$-thick transverse sections. Great care was taken to ensure that the cryosectioned muscles were cut along the transverse plane. All tissues were stained for capillarity using the lead nitrate-ATPase method described by Rosenblatt et al. (1987). A light microscope (Nikon Eclipse 80i; Nikon Instruments Inc.) was used to digitally acquire (Photometrics CoolSnap ES; Photometrics, Tucson, AZ, USA) $\times 20$ images of sectioned muscles in a checkerboard fashion across the entire muscle. Capillary and myofibre counting was performed by a single individual, who was blinded to the identity of each of the samples. For the gastrocnemius muscle, we obtained images in a checkerboard fashion across the entire muscle, thus both superficial and deep regions within the gastrocnemius were included in the analysis. For the plantaris and soleus muscles, the entire muscle was imaged and analysed. Counting was performed by visualization from acquired images using a custom program in MATLAB 
Table 1. Body, heart and hindlimb muscle mass (means $\pm \mathrm{SEM}$ )

\begin{tabular}{|c|c|c|c|c|}
\hline & Control & MM & Non-MM & $\begin{array}{c}P \text { value for } \\
\text { three-group comparison }\end{array}$ \\
\hline Body mass (g) & $32.8 \pm 0.56$ & $29.9 \pm 0.83^{*} \dagger$ & $32.5 \pm 0.55$ & 0.01 \\
\hline Heart mass (mg) & $152.4 \pm 3.18$ & $161.2 \pm 4.81$ & $154.5 \pm 3.04$ & 0.24 \\
\hline Heart mass/body mass ( $\left.\mathrm{mg} \mathrm{g}^{-1}\right)$ & $4.65 \pm 0.11$ & $5.39 \pm 0.08^{*} \dagger$ & $4.76 \pm 0.12$ & $<0.01$ \\
\hline Gastrocnemius mass (mg) & $123.9 \pm 4.23$ & $59.2 \pm 2.08^{*} \dagger$ & $134.3 \pm 3.22$ & $<0.01$ \\
\hline Gastrocnemius mass/body mass ( $\mathrm{mg} \mathrm{g}^{-1}$ ) & $3.78 \pm 0.08$ & $1.98 \pm 0.05^{*} \dagger$ & $4.13 \pm 0.08^{*}$ & $<0.01$ \\
\hline Plantaris mass (mg) & $14.2 \pm 0.53$ & $9.8 \pm 0.62 * \dagger$ & $12.9 \pm 0.35$ & 0.01 \\
\hline Plantaris mass/body mass ( $\mathrm{mg} \mathrm{g}^{-1}$ ) & $0.43 \pm 0.03$ & $0.33 \pm 0.03^{*}$ & $0.40 \pm 0.03$ & 0.049 \\
\hline Soleus mass (mg) & $7.6 \pm 0.93$ & $9.8 \pm 0.93^{*} \dagger$ & $6.4 \pm 1.04$ & $<0.01$ \\
\hline Soleus mass/body mass ( $\mathrm{mg} \mathrm{g}^{-1}$ ) & $0.23 \pm 0.02$ & $0.51 \pm 0.02 * \dagger$ & $0.20 \pm 0.01$ & $<0.01$ \\
\hline
\end{tabular}

Student's unpaired $t$ tests indicate ${ }^{*} P<0.05$ compared with control line; and $\uparrow P<0.05$ compared with Non-MM line.

(version 7.0.0.27; The Mathworks, Natick, MA, USA), allowing the operator to visually mark and count the capillaries and fibres on each image. Capillary-to-fibre ratio (C:F; number of capillaries/number muscle fibres), capillary density (CD; number of capillaries/muscle fibre area) and fibre cross-sectional area (FCSA) were separately calculated for the gastrocnemius, soleus and plantaris from a minimum of 175 images per mouse line.

\section{Protein analysis}

The GA, SOL and PLT muscles from each group were separately homogenized in a lysis buffer containing $50 \mathrm{~mm}$ Tris- $\mathrm{HCl}$ (pH 7.4), $150 \mathrm{~mm} \mathrm{NaCl}, 0.5 \%$ Triton X-100 and protease inhibitors (Complete ${ }^{\mathrm{TM}}$ Tablet; Roche Applied Science, Indianapolis, IN, USA). Homogenates were centrifuged at $4^{\circ} \mathrm{C}, 8000 \mathrm{~g}$ for $10 \mathrm{~min}$, and supernatants removed and placed in new tubes. Total protein was measured by the bicinchoninic acid method (BCA protein assay kit; Bio-Rad laboratories, Hercules, CA, USA).

Quantification of VEGF was made from a total of $100 \mu \mathrm{g}$ of protein using a commercially available ELISA kit according to the manufacturer's instructions (Model MMV00; R\&D Systems, Minneapolis, MN, USA).

Denatured samples $(20 \mu \mathrm{g})$ from GA and PLT muscles were separated on a 3-8\% SDS-PAGE and blotted onto a $0.45 \mu \mathrm{m}$ nitrocellulose membrane (Pierce nitrocellulose membrane; Thermo Scientific, Rockford, IL, USA). After blocking with 5\% fat-free milk, membranes were probed using antibodies against TSP-1 (1:250 dilution; clone A6.1, no. 399300; Invitrogen, Burlington, ON, Canada), $\beta$-tubulin (1:1000 dilution; no. 2148; Cell Signaling, Danvers, MA, USA), horseradish peroxidaseconjugated anti-mouse (1:1000 dilution; no.p0260; Dako, GE Healthcare, Piscataway, NJ, USA) and horseradish peroxidase-conjugated anti-rabbit (1:1000 dilution; no.p0217; Dako). Proteins were visualized using chemiluminescence detection (Pierce ECL; Thermo Scientific) and digitally imaged (G:BOX Gel imager; Syngene, Cambridge, UK). Images were captured using Genesnap software (version 7.01; Syngene). Equal protein loading was verified by immunodetection of $\beta$-tubulin as our loading control. Quantification of protein expression levels were carried out using NIH ImageJ Software (version 1.62) and expressed as densitometric arbitrary units (a.u.). The TSP-1 expression was normalized to $\beta$-tubulin expression level. Owing to insufficient sample volume, we were not able to run Western analysis of TSP-1 on the SOL muscle.

\section{Statistics}

All data are presented as means \pm SEM. To examine body mass, organ masses and muscle capillarity (measured only in basal conditions), we used a one-way ANOVA comparing the three lines of mice. To examine protein expression, we used a two-way ANOVA comparing the three lines of mice in basal conditions and in response to acute exercise, while also testing for an interaction between line and exercise condition. Separate analyses were performed for each of the three muscles studied (StatView, 5.0.1). When a main effect was observed, post hoc testing was performed using Student's unpaired $t$ test. An $\alpha$ level of $P<0.05$ was selected for statistical significance.

\section{Results}

\section{Body and muscle masses}

Body mass differed significantly among lines $(P=0.006)$. As seen in Table 1, MM mice had 9\% lower body mass than Non-MM mice $(P=0.008)$ and $10 \%$ lower than control mice $(P=0.003)$. The MM mice also had a $16 \%$ larger heart mass (per gram body mass) compared with control line mice $(P<0.001)$ and a $13 \%$ increase over the NonMM line $(P<0.001)$. There was no statistical difference in relative heart mass between the Non-MM and the control animals.

All three muscles of the triceps surae differed significantly in mass among all lines (GA, $P<0.001$; SOL, $P<0.01$; and PLT, $P<0.05)$, even when expressed relative to body mass (GA, $P<0.001$; SOL, $P<0.001$; and PLT, $P<0.05$; Table 1). The MM mice had a $56 \%$ decrease in 
GA mass compared with Non-MM line $(P<0.001)$ and a $52 \%$ reduction compared with control mice $(P<0.001)$. The Non-MM line also had $8 \%$ greater GA mass than control mice $(P=0.036)$. On a mass-specific basis, the MM mice had a $48 \%$ reduction compared with the control mice $(P<0.001)$ and a 52\% reduction compared with the Non-MM line $(P<0.001)$. The Non-MM line also had an $8 \%$ increase when corrected for body mass compared with the control mice $(P<0.001)$. As expected (Guderley et al. 2008; Wong et al. 2009), superficial portions of the GA muscle in MM mice showed the distinct appearance of many small fibres not present in either of the other lines (Fig. 1).

The PLT of the MM mouse was also 32\% smaller than in the Non-MM line $(P=0.039)$ and $45 \%$ smaller than in control mice $(P=0.004)$. There was no difference between the Non-MM line and control mice. On a mass-specific basis, only the MM mice and control mice showed any statistical difference, with a $30 \%$ reduction in the MM PLT mass $(P=0.015)$ compared with the control animals.

The SOL of MM mice was 53\% larger than in the NonMM mice $(P<0.001)$ and $29 \%$ larger than in the control mice $(P=0.005)$. There was no statistical difference between the Non-MM line and the control mice. On a mass-specific basis, the MM line had $155 \%$ greater SOL mass than the Non-MM line $(P<0.001)$ and a $122 \%$ greater mass than the control line $(P<0.001)$. There was no difference between the Non-MM line and the control animals after correcting for body mass.

\section{Morphometry}

There was a significant main effect for C:F, CD and FCSA among all groups for the GA (all $P<0.001$ ) and PLT (all $P<0.05)$, but not for the SOL muscle. In the GA, MM mice had a $25 \%$ greater C:F and $43 \%$ greater $\mathrm{CD}$ than the Non-MM line $(P<0.001)$ and $38 \%$ greater C:F and $46 \%$ greater $\mathrm{CD}$ than the control mice $(P<0.001 ;$ Fig. 2$)$. There was no difference in C:F or CD between the Non-MM and control mice for the GA. The GA FCSA was decreased by 39 and $36 \%$ in the MM mice compared to Non-MM and control mice, respectively $(P<0.001$; Fig. 2$)$.

In the PLT, there was no difference in C:F, CD and FCSA between MM mice and the Non-MM line (Fig. 2); however, the MM mice and Non-MM mice had a 35 $(P=0.002)$ and $27 \%(P=0.005)$ greater $\mathrm{C}: \mathrm{F}$, and a 24 $(P<0.034)$ and $31 \%(P<0.003)$ greater CD in the PLT compared with control mice (Fig. 2).

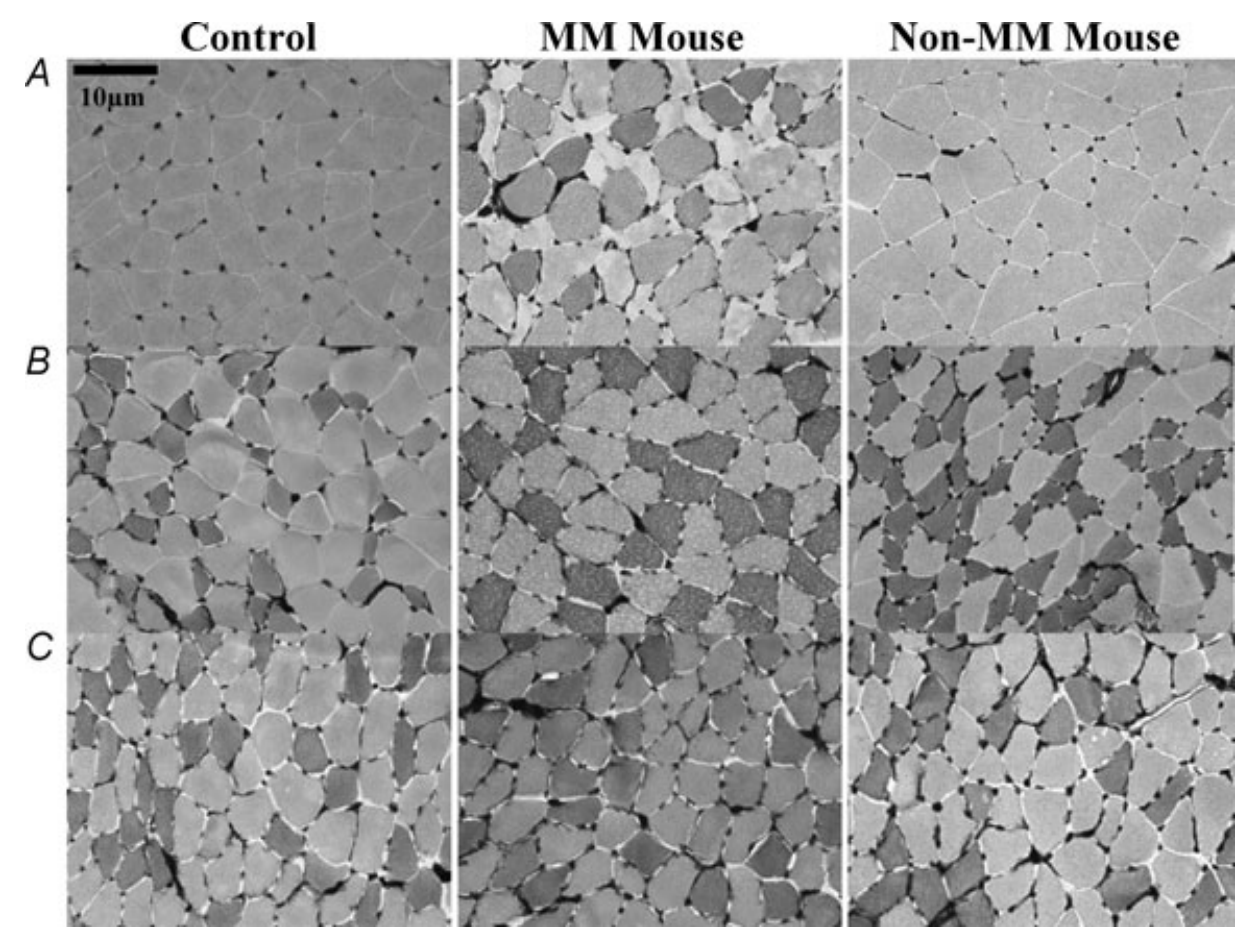

Figure 1. Representative images of triceps surea muscles

$A$, superficial gastrocnemius. $B$, soleus. $C$, plantaris. Capillaries were stained using lead-ATPase appear as black dots outside the muscle. The presence of very small muscle fibres in the gastrocnemius muscle $(A)$ is evident in MM mice compared with control and Non-MM mice. Capillary-to-fibre ratio is significantly elevated in gastrocnemius muscle of MM mice $(A)$ compared with control and Non-MM mice $(P<0.001)$, and significantly greater in plantaris muscle of MM mice $(B)$ compared with control mice $(P<0.001)$, but not Non-MM mice. No difference in soleus muscle capillarity was observed between the groups (C). 
Table 2. Muscle fibre-type composition

\begin{tabular}{|c|c|c|c|c|c|c|c|c|}
\hline & \multicolumn{4}{|c|}{ Type I (\%) } & \multicolumn{4}{|c|}{ Type II (\%) } \\
\hline & Control & MM & Non-MM & $P$ value & Control & MM & Non-MM & $P$ value \\
\hline Gastrocnemius (deep) & $20 \pm 4$ & $30 \pm 4 \dagger$ & $24 \pm 3$ & 0.18 & $79 \pm 4$ & $70 \pm 4 \dagger$ & $75 \pm 3$ & 0.15 \\
\hline Gastrocnemius (superficial) & $2 \pm 1$ & $12 \pm 3^{*}$ & $7 \pm 3$ & 0.04 & $98 \pm 1$ & $89 \pm 3^{*}$ & $95 \pm 2$ & 0.02 \\
\hline Plantaris & $38 \pm 6$ & $38 \pm 1$ & $39 \pm 2$ & 0.96 & $62 \pm 6$ & $62 \pm 2$ & $61 \pm 2$ & 0.91 \\
\hline Soleus & $40 \pm 2$ & $32 \pm 5$ & $39 \pm 4$ & 0.32 & $61 \pm 3$ & $68 \pm 5$ & $61 \pm 3$ & 0.29 \\
\hline
\end{tabular}

$P$ value represents ANOVA comparison among three groups; $* P<0.05$ compared with control line; and $\dagger P=0.07$ compared with control line.

There was not a significant main effect for C:F, CD or FCSA in the SOL (data not shown).

There was a significant main effect for fibre type for the superficial GA between the mouse lines $(P<0.05)$. Post hoc analysis revealed that $\mathrm{MM}$ mice had $86 \%$ more type I fibres $(P=0.014)$ and $10 \%$ fewer type II fibres $(P=0.007)$ than control mice (Table 2). In the deep GA, a significant main effect for fibre type was not found between the mouse lines (type I, $P=0.179$; and type II, $P=0.154$ ); however, this region of the GA exhibited a similar trend towards having an increase in the proportion of type I and decrease in proportion of type II myofibres in MM compared with control mice. There was no significant difference between MM mice and Non-MM mice in either portion of the GA. No significant differences in fibre type between the mouse lines in either the PLT or SOL were observed.

\section{Protein expression of VEGF}

Basal levels. There was a main effect among mouse lines for basal VEGF expression in the GA $(P=0.017)$ and the PLT muscles $(P=0.031)$. In the GA, MM mice had a $58 \%$ higher basal expression of VEGF compared with control mice $(P=0.006$; Fig. 3$)$. Although basal VEGF expression was also $33 \%$ greater in Non-MM compared with control mice, this difference did not reach statistical significance $(P=0.055)$. There was no significant difference in basal VEGF expression between MM and Non-MM mice.

In the PLT muscle, we observed $79 \%$ greater VEGF expression in $\mathrm{MM}$ mice compared with control mice $(P=0.010)$, but no significant difference between MM and Non-MM mice $(P=0.081)$. There was also no significant difference between Non-MM mice and control mice, despite a similar tendency for $28 \%$ greater VEGF levels in Non-MM mice $(P=0.251)$, like that seen in the gastrocnemius.

No significant main effect for basal VEGF expression was observed in the SOL muscle (data not shown).

Response to acute exercise. There was a main effect for VEGF response among mouse lines $(P=0.0003)$ and a significant exercise $\times$ line interaction in the GA $(P=0.047$; Fig. 3$)$. Only the MM mice showed a significant VEGF response to acute exercise, resulting in
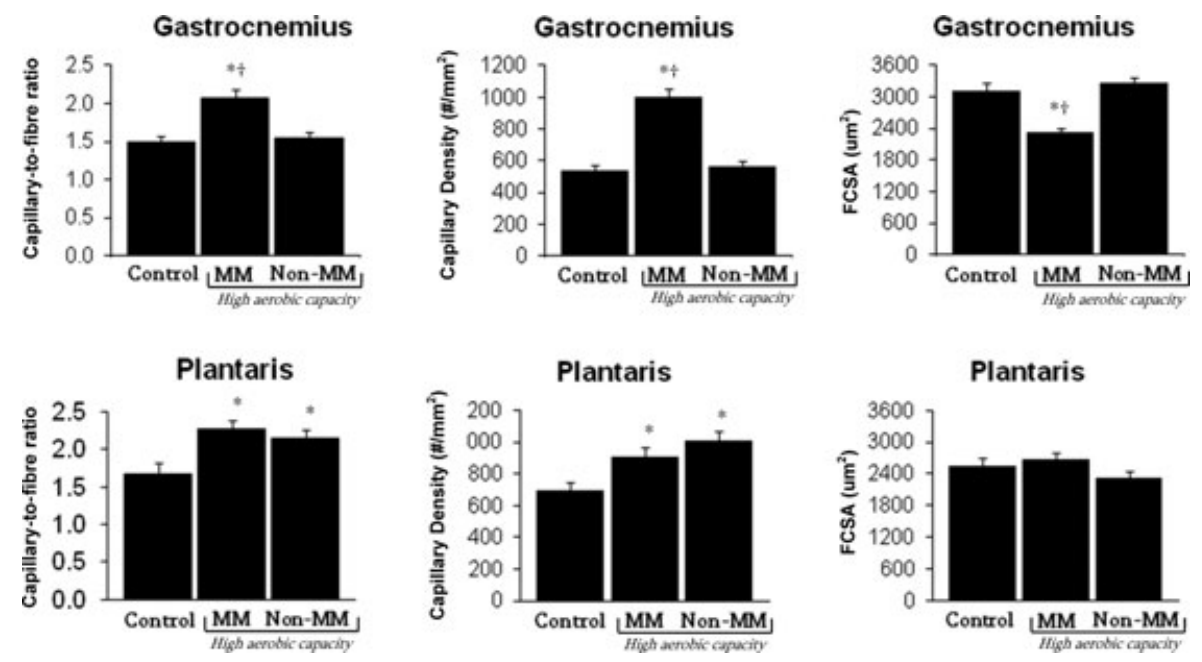

Figure 2. Capillary-to-fibre ratio (C:F), capillary density (CD) and fibre cross-sectional area (FSCA) of the triceps surae muscles

In the gastrocnemius, MM mice had an increase in C:F and CD and a decrease in FCSA compared with both control and Non-MM mice. In plantaris, MM and Non-MM had an increased C:F and CD compared with control mice. ${ }^{*} P<0.05$ compared with control mice; and $\uparrow P<0.05$ compared with Non-MM mice. 
a $41 \%$ increase in VEGF in the GA from basal levels $(P=0.009)$. The control mice had a very similar $38 \%$ increase in VEGF from baseline, but this did not reach statistical significance $(P=0.081)$, which was probably due to the greater variability observed in the VEGF response to exercise in these mice. Surprisingly, there was no VEGF response to exercise in the Non-MM line GA.

There was no statistically significant interaction between the VEGF response to exercise and line in either of the other muscles studied (i.e. PLT and SOL). However, similar to the GA, VEGF in the PLT increased $28 \%$ in response to exercise in $\mathrm{MM}$ mice $(P=0.038)$. Non-MM mice showed a non-significant increase $(21 \% ; P=0.158)$ for VEGF in the plantaris, while control mice did not respond to exercise.

No significant difference between groups for the VEGF response to exercise was observed in the SOL muscle (data not shown).
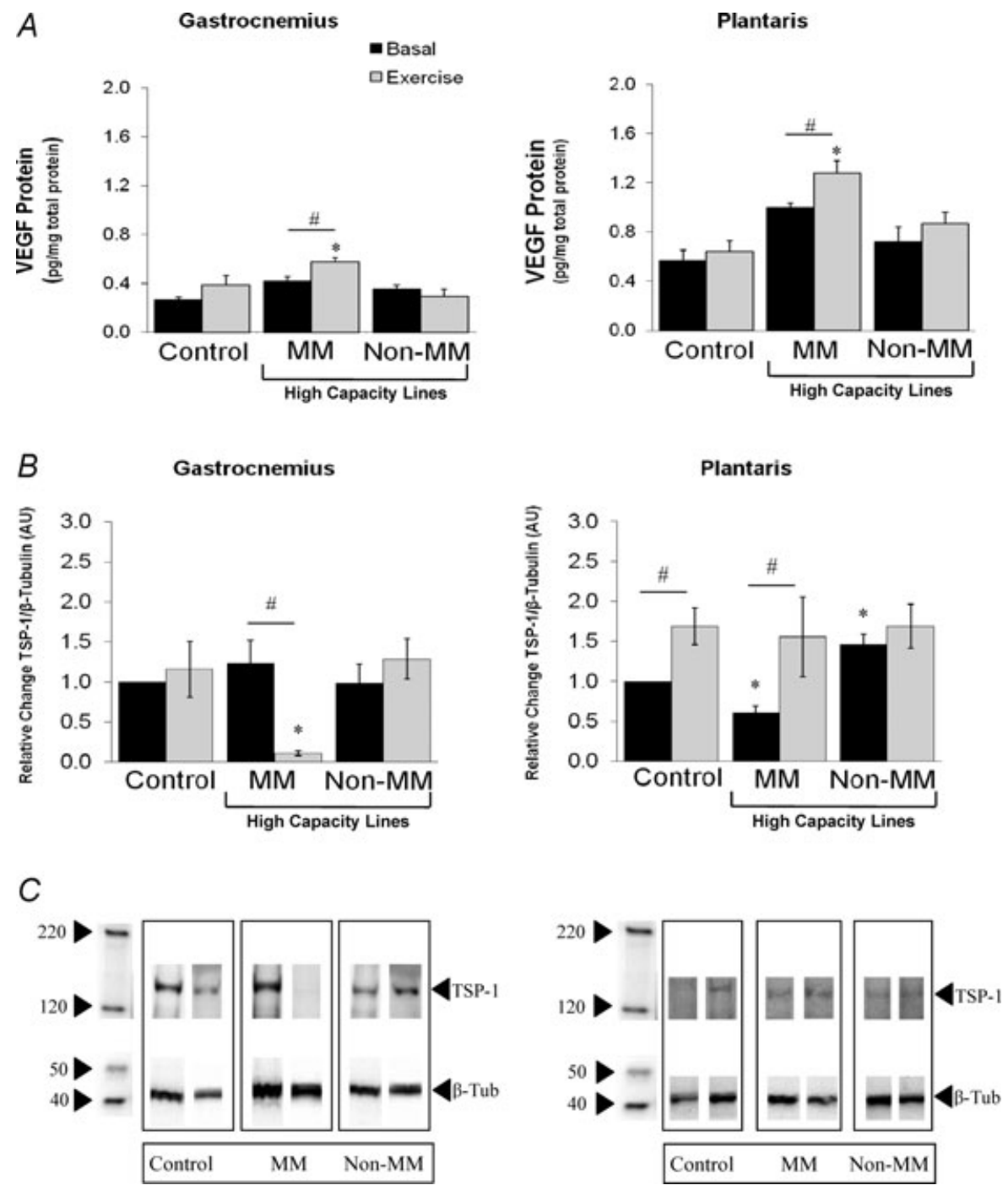

Figure 3. Comparison of basal and postexercise levels of vascular endothelial growth factor (VEGF) and thrombospondin-1 (TSP-1) in the triceps surae muscles

$A$, there was a statistically significant interaction between acute exercise and VEGF levels in the gastrocnemius (GA) and plantaris (PLT). In both muscles, MM mice had increased basal levels of VEGF compared with control animals, and a significant VEGF response to acute exercise. The VEGF levels did not differ between the Non-MM and control mice in either muscle, although there was a trend for an increase in the GA $(P=0.055)$. $B$, there was a statistically significant interaction between acute exercise and TSP-1 levels in the GA and PLT. In basal conditions in the PLT, the MM mice had lower TSP-1 levels than either the control or Non-MM mice, and in the GA and PLT the MM mice had a significant TSP-1 response to acute exercise. The Non-MM mice had significantly higher basal levels of TSP-1 in the PLT than control mice. Also in the PLT, control animals had a significant TSP-1 response to acute exercise. $C$, representative Western blots from TSP-1 analysis $(B) .{ }^{*} P<0.05$ compared with control mice, same exercise state (basal or acute exercise); and \#P<0.05 compared with basal levels. 


\section{Protein expression of TSP-1}

Basal levels. Basal levels of TSP-1 in the GA muscle were not different among mouse lines. However, a significant main effect for basal TSP-1 expression in the PLT was observed among mouse lines. In the PLT, MM mice had 39\% lower TSP-1 levels compared with control mice $(P<0.01)$ and $58 \%$ lower compared with the Non-MM mice $(P<0.01$; Fig. 3$)$. Non-MM mice exhibited a $46 \%$ increase in basal TSP-1 compared with control mice $(P<0.001$; Fig. 3$)$.

Response to acute exercise. There was a significant exercise $\times$ line interaction in the GA $(P=0.014$; Fig. 3$)$. In the GA, control mice and Non-MM mice showed no statistical TSP-1 response to acute exercise, whereas MM mice exhibited a 90\% decrease in TSP-1 $(P=0.003)$. In the PLT, there was also a significant effect for TSP-1 in response to acute exercise $(P=0.005)$, where TSP-1 expression increased by $47 \%(P<0.01)$ in control mice and by $55 \%(P<0.05)$ in MM mice (Fig. 3$)$. There was no significant acute exercise response in Non-MM mice (Fig. 3).

\section{Discussion}

The main finding of this study is that elevated skeletal muscle capillarity in MM mice bred for high aerobic capacity is associated with an altered balance between VEGF and TSP-1 protein expression (Fig. 3). These data are consistent with the observations that VEGF and TSP-1 are essential regulators of the skeletal muscle microvascular bed. Surprisingly, in Non-MM mice a similar diverging correlation between VEGF and TSP1 in relation to changes in muscle capillarity was not found in the PLT muscle. In general, however, these data support the notion that VEGF and TSP-1 are key physiological regulators of angiogenesis within skeletal muscle. Moreover, when comparing the basal expression of these factors in MM mice against control mice, these are among the first data to suggest that muscle capillarity and the capacity for exercise-induced angio-adaptation may, in part, be accounted for by inherited traits related to the expression of VEGF, and possibly TSP-1, within skeletal muscle.

\section{Basal expression of VEGF and TSP-1 correlates with elevated skeletal muscle capillarity in MM mice, but not in Non-MM mice}

In this study, we report that MM mice have increased capillarity in the both the GA and the PLT (Fig. 2), which is associated with elevated basal expression of VEGF in each muscle (Fig. 3). At the same time, we observed no difference in basal skeletal muscle TSP-1 expression in the GA, but a significant decrease in the PLT (Fig. 3) which, when combined with the elevated expression of VEGF in both GA and PLT, is consistent with a proangiogenic environment within the muscle. In contrast, neither capillarity nor VEGF expression was different in the SOL muscle of MM or Non-MM mice compared with control mice.

In Non-MM mice, which are also high aerobic capacity mice but without the unique 'mini-muscle' phenotype, we found that only the PLT had elevated capillarity, whereas neither GA nor SOL muscle capillarity was different compared with control animals (Fig. 2). Although there was a trend for elevated basal VEGF expression in the PLT of Non-MM mice $(P=0.07)$, we also found elevated basal TSP-1 expression $(P<0.01)$, which together could be argued as an anti-angiogenic state rather than pro-angiogenic. Nonetheless, an increase in PLT muscle capillarity was observed in Non-MM mice (Fig. 2). In contrast, no significant changes in basal VEGF were seen in either the GA or SOL muscles of Non-MM mice. Likewise, TSP-1 was not different in GA muscle, and no differences in capillarity were seen in either the GA or the SOL of Non-MM mice compared with control animals.

Taken together, the elevated muscle capillarity in the GA of MM mice is most probably explained by elevated basal VEGF. In the PLT, elevated muscle capillarity occurred in the presence of decreased basal TSP-1 expression and little or no increase in VEGF, suggesting that TSP-1 may also be playing an important role, at least in the PLT of MM mice. Despite the differences in these individual muscles, there is growing evidence that physiological regulation of angiogenesis is dependent on the balance between positive and negative angiogenic proteins such that the ratio of VEGF to TSP-1 expression may actually be more important than their individual expression levels per se (Olfert \& Birot, 2011). The present results support the notion of an altered balance leading to capillary adaptation, with the notable exception of the PLT muscle in Non-MM mice. One explanation for this discrepancy may be that other angiogenic regulators (such as endostatin, angiopoetins and their receptors, matrix metalloproteinases) are also playing a role, but are not accounted for in this study. Indeed, while we focused on VEGF and TSP-1 because of their recognized prominence in effecting changes in the skeletal muscle vascular bed, it is entirely possible (and likely) that other regulators also contribute to the basal angiogenic state (Olfert \& Birot, 2011).

It is interesting to note that increased capillarity occurred predominately in skeletal muscle composed of glycolytic myofibres (i.e. GA and PLT) and not in muscle composed of mostly oxidative myofibres (i.e. SOL). This observation is consistent in both high aerobic selected lines (i.e. MM and Non-MM), and might suggest that muscles 
with greater glycolytic metabolic profiles experienced the greatest amount of evolutionary change in these selectively bred lines of mice. This idea is consistent with the observation that glycolytic myofibres may be principally responsible for driving the angiogenic response to acute exercise (Birot et al. 2003; Waters et al. 2004). It may also be that muscles having a predominant role in locomotor activity (i.e. GA and PLT) would be the most evolved in these mice, whereas postural skeletal muscles (such as the SOL) are less likely to have been influenced.

\section{Exercise capacity, angiogenesis and VEGF}

It remains clear that muscles found to have elevated basal VEGF expression are also the muscles with elevated capillarity (Fig. 4). In TSP-1 knockout mice, increases in skeletal muscle capillarity (achieved without exercise training) are also associated with greater exercise capacity (Malek \& Olfert, 2009), whereas losses in muscle capillarity (due to deletion of VEGF in myocytes) are shown to reduce exercise performance (Olfert et al. 2009). The strong association between exercise capacity and muscle capillarity is also supported in selectively bred rats with high and low aerobic capacity (Howlett et al. 2003). Thus, the present findings are consistent with the evidence that skeletal muscle capillarity and muscle $\mathrm{O}_{2}$ conductance are critical determinants of aerobic exercise performance (Howlett et al. 2003). It is interesting to note, however, that $\dot{V}_{\mathrm{O}_{2} \text { max }}$ is not statistically different between MM mice and any of the other high aerobic capacity mouse lines, except during exercise in hypoxia (Rezende et al. 2006a,b). If $\mathrm{O}_{2}$ transport in the mouse is principally limited at the muscle-capillary interface, as implied by these and the other aforementioned data, then the greater capillarity in GA muscle of MM mice could help explain the advantage in $\dot{V}_{\mathrm{O}_{2} \max }$ seen during hypoxic exercise over Non-MM mice (Rezende et al. 2006a). Although these data do not establish a cause-and-effect relationship, they are in harmony with the view that VEGF-mediated increases in skeletal muscle capillarity contribute to the greater exercise capabilities observed in mice with an innate high aerobic capacity. Given the positive relationship noted for VEGF and C:F (Fig. 4) and the notable absence of an inverse relationship for TSP-1 and C:F (Fig. 4), it could be argued that VEGF may be the most critical determinant for muscle capillarity. More importantly, the fact that VEGF and TSP1 had different responses within the triceps surae muscles between these two high aerobic capacity lines (i.e. MM
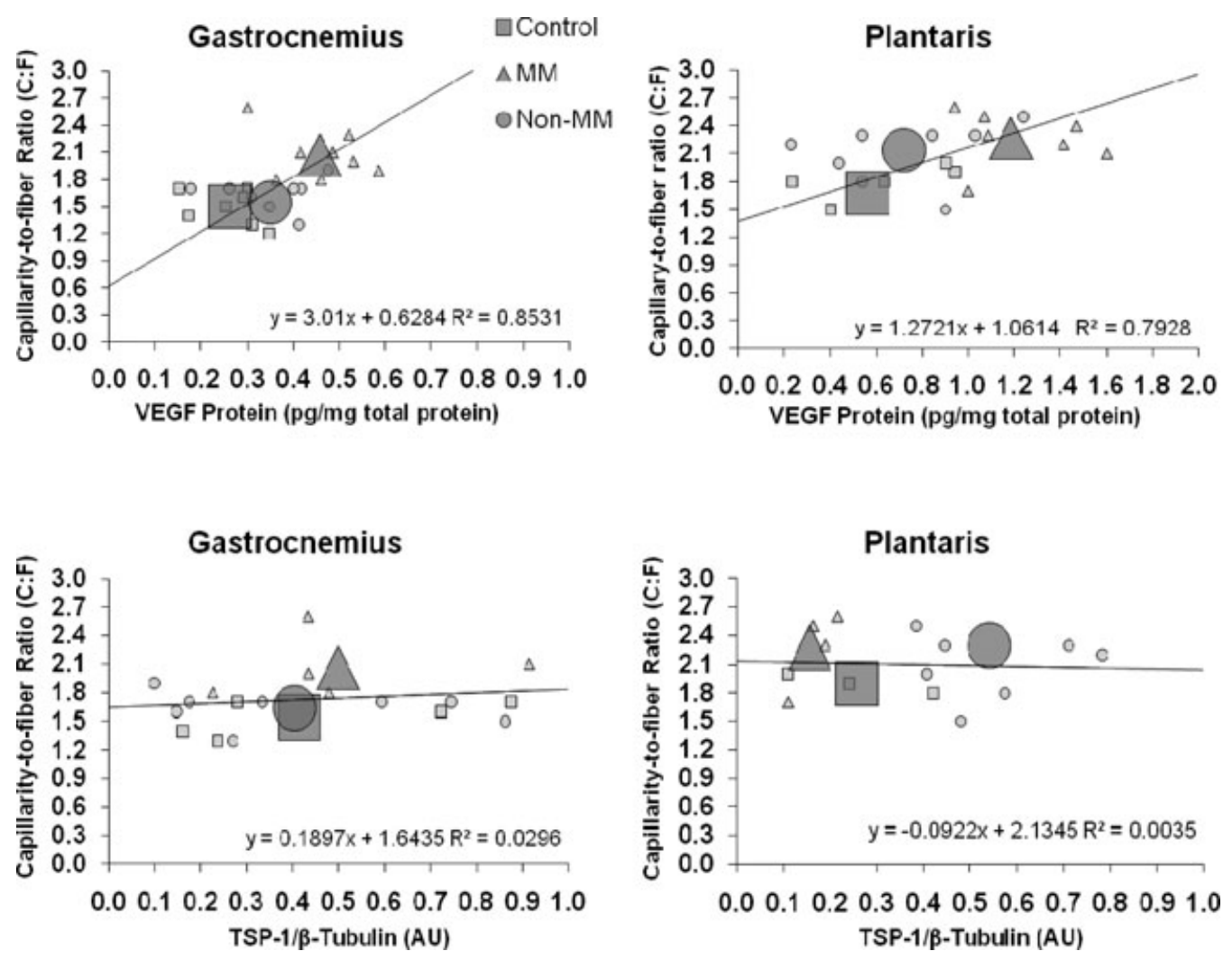

Figure 4. Correlation of muscle VEGF and TSP-1 levels to capillary-to-fibre ratio (C:F) Least-squares linear regression of C:F on basal VEGF and TSP-1 levels in the GA and PLT muscles of the three mouse lines. Large symbols indicate mean values for each line and small symbols are values for individual animals. Squares indicate control mice, triangles MM mice and circles Non-MM mice. 
versus Non-MM) suggests that basal angiogenic protein expression may be, at least in part, an inherited trait that may also account for some of the biological variability in exercise capacity.

\section{Genetic background may influence angiogenic responses to acute exercise}

In the context of exercise training, it has been shown in standard laboratory mice and rats that the VEGF response to acute exercise is attenuated in skeletal muscle post-training (Olfert et al. 2001; Malek \& Olfert, 2009). It has been postulated that this is a direct response to training-induced angio-adaptation (i.e. increased muscle capillarity, improved blood flow distribution to working muscle, and increased $\mathrm{O}_{2}$ conductance into the myocyte) that functions to relieve metabolic stress on the myocyte that is imposed by exercise. Given that local tissue hypoxia is thought to be among the key stimuli for the VEGF response to exercise (Levy et al. 1995; Breen et al. 1996; Richardson et al. 1999; Olfert et al. 2001), we have previously speculated that the attenuated VEGF response to an acute bout of exercise post-training is the result of increased muscle capillarity and greater $\mathrm{O}_{2}$ conductance during exercise (Olfert et al. 2001; Malek \& Olfert, 2009). The present data could be interpreted to suggest that a mechanism other than local hypoxia may be involved in the modulation of VEGF during acute responses to exercise. Indeed, if increased capillarity reduces local tissue hypoxia, and this is responsible for modulating the VEGF response, then it would be expected that an attenuated VEGF response to exercise would be seen in MM mice (which inherently displayed a greater number of capillaries in their muscle compared with normal mice). However, as it has also previously been shown that MM mice have increased aerobic metabolic enzyme activities in hindlimb muscles (Guderley et al. 2008), it could also be that MM mice simply have a much higher oxygen flux and thus the level of $\mathrm{O}_{2}$ within the muscles is relatively unchanged compared with Non-MM and control mice (despite the greater number of muscle capillaries). We also cannot exclude the possibility that other mechanisms, such as shear stress, local drops in muscle $\mathrm{pH}$, modulation of other matrix regulators and/or other angiogenic factors, may have indirect influences on the VEGF response to exercise.

Some support for modulation of other angiogenic factors comes from the TSP-1 data in the present study. Thrombospondin-1 is a potent inhibitor of angiogenesis and of VEGF directly (Gupta et al. 1999; Greenaway et al. 2007). In the MM mice, we found that TSP-1 levels were lower in the PLT in basal conditions and in the GA in the acute exercise conditions (Fig. 3). In both of these circumstances, VEGF is also increased. This supports the notion of an altered balance between positive (e.g. VEGF) and negative (e.g. TSP-1) angiogenic factors (Hanahan \&
Folkman, 1996; Olfert \& Birot, 2011). This altered balance would be another elegant way that the MM mice are genetically more able to adapt to an exercise stimulus.

Another potential explanation for the greater VEGF response to exercise in $\mathrm{MM}$ mice may be that the VEGF response to exercise is also genetically influenced. Although it is difficult to decipher the relative contribution of genetic versus environmental influences in setting an individual's exercise capacity, evidence supporting the genetic influences can be seen from a recent report involving fine genotype mapping of MM mice (Hartmann et al. 2008). Hartmann et al. (2008) localized the gene responsible for the mini-muscle phenotype to a $2.6335 \mathrm{Mb}$ of MMU11, a region that harbours $\sim 100$ expressed or predicted genes, many with known roles in muscle development and/or function. Upon examination of the genes within this region, we have identified two genes, Tnfsf12/TWEAK and Ephrin ligands/receptors, both of which have been shown to have some regulatory role in angiogenesis and/or endothelial cell proliferation (Helbling et al. 2000; Cheng et al. 2002; Donohue et al. 2003). What role, if any, these factors may exert on skeletal muscle VEGF remains to be determined, but it is tempting to speculate that these (or other) genes may support and/or enhance angiogenesis in MM mice.

It should be noted that our data are not the first to suggest that the VEGF response to exercise may be influenced by genetic background. Prior et al. (2006) have shown that genetic variants in the promoter region of the VEGF gene correlate with whole-body $\dot{V}_{\mathrm{O}_{2} \max }$ in humans. Similar results were also reported in response to hypoxia-mediated increased in VEGF gene expression in cultured myotubes (Prior et al. 2006). Our data, combined with these previous studies, imply that individual gene responses may influence exercise performance, and suggest that the VEGF response to exercise may, at least in part, be an inherited trait.

\section{Comparison of muscle mass and fibre-type profile among mouse lines}

It has previously been shown that MM mice have a significantly decreased triceps surae muscle mass (Syme et al. 2005; Guderley et al. 2006, 2008; McGillivray et al. 2009). Likewise, the present study shows that GA and PLT muscle mass was decreased in the MM mice when compared with the control and Non-MM mice (Table 1). Opposite to what is seen in the GA and PLT muscles, the SOL of the MM mice was increased over $130 \%$ (on a body mass-specific basis) when compared with both the control mice and the Non-MM line (Table 1).

In mice, the GA has an aerobic profile that is mixed, with superficial portions of the muscle containing mainly type IIb/x fibres, whereas the deep portions contains many type IIa and type I fibres (Guderley et al. 2008; Wong 
et al. 2009). In contrast, the SOL is comprised primarily of type I or IIa fibres. Assessment of fibre type in these mice reveals that MM mice have a greater proportion of type I than type II myofibres compared with control and Non-MM mice (Table 2). These findings are supported by earlier observations that MM mice have increased type I and type IIa fibres along with decreased type IIb fibres in the medial GA muscle (Guderley et al. 2008). This myofibre composition is consistent with the elevated C:F ratio found in the GA of the MM mice in the present study, further supporting the notion of a greater oxidative muscle metabolic profile capable of supporting greater aerobic capacity.

This also raises the issue of the influence of muscle mass and/or fibre type/size on skeletal muscle aerobic performance. It could be argued that greater cardiac output (as indicated by larger cardiac mass in both absolute terms and relative to body size) could itself contribute to improved exercise capacity in MM mice (Table 1). Perhaps the strongest argument against this hypothesis is provided by the Non-MM mice, which exhibit an equally high aerobic endurance capacity to the MM mice but do not have greater cardiac mass (in absolute or relative terms) compared with control animals (Garland et al. 2002; Table 1). Moreover, we would also contend (irrespective of any improvements in central cardiac function) that blood flow to the working muscle is also controlled by local metabolic demand. In combination with the greater muscle metabolic potential of MM mice, such as increased mitochondrial enzymatic activity (Guderley et al. 2006), it is perhaps debatable whether greater cardiac function in these MM mice might be considered as improved or simply an attempt to keep up with metabolic demand of the working muscle. Nonetheless, there are presently no data which document cardiac output or function in these mice, thus we do not know if cardiac function is altered.

In summary, these data generally support the notion that skeletal muscle angiogenesis in physiological conditions is likely to be controlled by a balance between pro- and anti-angiogenic factors, and also suggest that the expression of VEGF versus TSP-1 may differ among muscles. Moreover, the observation that basal VEGF and TSP-1 expression, and the VEGF and TSP-1 response to exercise, were different between the two high aerobic capacity mouse lines seems to suggest first, that a mechanism other than local hypoxia might be regulating the VEGF response to exercise and second, that the VEGF response to exercise may, in part, be genetically determined.

\section{References}

Andersen P \& Henriksson J (1977). Capillary supply of the quadriceps femoris muscle of man: adaptive response to exercise. J Physiol 270, 677-690.
Birot OJ, Koulmann N, Peinnequin A \& Bigard XA (2003). Exercise-induced expression of vascular endothelial growth factor mRNA in rat skeletal muscle is dependent on fibre type. J Physiol 552, 213-221.

Breen EC, Johnson EC, Wagner H, Tseng H-M, Sung LA \& Wagner PD (1996). Angiogenic growth factor mRNA responses in muscle to a single bout of exercise. J Appl Physiol 81, 355-361.

Cheng N, Brantley DM \& Chen J (2002). The ephrins and Eph receptors in angiogenesis. Cytokine Growth Factor Rev 13, 75-85.

Donohue PJ, Richards CM, Brown SA, Hanscom HN, Buschman J, Thangada S, Hla T, Williams MS \& Winkles JA (2003). TWEAK is an endothelial cell growth and chemotactic factor that also potentiates FGF-2 and VEGF-A mitogenic activity. Arterioscler Thromb Vasc Biol 23, 594-600.

Feder ME, Garland T Jr, Marden JH \& Zera AJ (2010). Locomotion in response to shifting climate zones: not so fast. Annu Rev Physiol 72, 167-190.

Garland T Jr, Kelly SA, Malisch JL, Kolb EM, Hannon RM, Keeney BK, Van Cleave SL \& Middleton KM (2011). How to run far: multiple solutions and sex-specific responses to selective breeding for high voluntary activity levels. Proc Biol Sci 278, 574-581.

Garland T Jr, Morgan MT, Swallow JG, Rhodes JS, Girard I, Belter JG \& Carter PA (2002). Evolution of a small-muscle polymorphism in lines of house mice selected for high activity levels. Evolution 56, 1267-1275.

Gavin TP \& Wagner PD (2001). Effect of short-term exercise training on angiogenic growth factor gene responses in rats. J Appl Physiol 90, 1219-1226.

Greenaway J, Lawler J, Moorehead R, Bornstein P, Lamarre J \& Petrik J (2007). Thrombospondin-1 inhibits VEGF levels in the ovary directly by binding and internalization via the low density lipoprotein receptor-related protein-1 (LRP-1). J Cell Physiol 210, 807-818.

Guderley H, Houle-Leroy P, Diffee GM, Camp DM \& Garland $\mathrm{T} \mathrm{Jr}$ (2006). Morphometry, ultrastructure, myosin isoforms, and metabolic capacities of the "mini muscles" favoured by selection for high activity in house mice. Comp Biochem Physiol B Biochem Mol Biol 144, 271-282.

Guderley H, Joanisse DR, Mokas S, Bilodeau GM \& Garland T $\mathrm{Jr}$ (2008). Altered fibre types in gastrocnemius muscle of high wheel-running selected mice with mini-muscle phenotypes. Comp Biochem Physiol B Biochem Mol Biol 149, 490-500.

Gupta K, Gupta P, Wild R, Ramakrishnan S \& Hebbel RP (1999). Binding and displacement of vascular endothelial growth factor (VEGF) by thrombospondin: effect on human microvascular endothelial cell proliferation and angiogenesis. Angiogenesis 3, 147-158.

Gustafsson T, Knutsson A, Puntschart A, Kaijser L, Nordqvist AC, Sundberg CJ \& Jansson E (2002). Increased expression of vascular endothelial growth factor in human skeletal muscle in response to short-term one-legged exercise training. Pflugers Arch 444, 752-759.

Hanahan D \& Folkman J (1996). Patterns and emerging mechanisms of the angiogenic switch during tumorigenesis. Cell 86, 353-364. 
Hartmann J, Garland T Jr, Hannon RM, Kelly SA, Muñoz G \& Pomp D (2008). Fine mapping of "mini-muscle," a recessive mutation causing reduced hindlimb muscle mass in mice. J Hered 99, 679-687.

Helbling PM, Saulnier DM \& Brandli AW (2000). The receptor tyrosine kinase EphB4 and ephrin-B ligands restrict angiogenic growth of embryonic veins in Xenopus laevis. Development 127, 269-278.

Henderson KK, Wagner H, Favret F, Britton SL, Koch LG, Wagner PD \& Gonzalez NC (2002). Determinants of maximal $\mathrm{O}_{2}$ uptake in rats selectively bred for endurance running capacity. J Appl Physiol 93, 1265-1274.

Houle-Leroy P, Guderley H, Swallow JG \& Garland T Jr (2003). Artificial selection for high activity favors mighty mini-muscles in house mice. Am J Physiol Regul Integr Comp Physiol 284, R433-R443.

Howlett RA, Gonzalez NC, Wagner HE, Fu Z, Britton SL, Koch LG \& Wagner PD (2003). Genetic models in applied physiology: skeletal muscle capillarity and enzyme activity in rats selectively bred for running endurance. J Appl Physiol 94, 1682-1688.

Howlett RA, Kirkton SD, Gonzalez NC, Wagner HE, Britton SL, Koch LG \& Wagner PD (2009). Peripheral oxygen transport and utilization in rats following continued selective breeding for endurance running capacity. J Appl Physiol 106, 1819-1825.

Koch LG \& Britton SL (2001). Artificial selection for intrinsic aerobic endurance running capacity in rats. Physiol Genomics $5,45-52$.

Koch LG, Britton SL, Barbato JC, Rodenbaugh DW \& DiCarlo SE (1999). Phenotypic differences in cardiovascular regulation in inbred rat models of aerobic capacity. Physiol Genomics 1, 63-69.

Koteja P, Garland T Jr, Sax JK, Swallow JG \& Carter PA (1999). Behaviour of house mice artificially selected for high levels of voluntary wheel running. Anim Behav 58, 1307-1318.

Lee S, Chen TT, Barber CL, Jordan MC, Murdock J, Desai S, Ferrara N, Nagy A, Roos KP \& Iruela-Arispe ML (2007). Autocrine VEGF signaling is required for vascular homeostasis. Cell 130, 691-703.

Levy AP, Levy NS, Wegner S \& Goldberg MA (1995). Transcriptional regulation of rat vascular endothelial growth factor gene by hypoxia. J Biol Chem 270, 13333-13340.

Lloyd PG, Prior BM, Yang HT \& Terjung RL (2003). Angiogenic growth factor expression in rat skeletal muscle in response to exercise training. Am J Physiol Heart Circ Physiol 284, H1668-H1678.

McGillivray DG, Garland T Jr, Dlugosz EM, Chappell MA \& Syme DA (2009). Changes in efficiency and myosin expression in the small-muscle phenotype of mice selectively bred for high voluntary running activity. J Exp Biol 212, 977-985.

Malek MH \& Olfert IM (2009). Global deletion of thrombospondin-1 increases cardiac and skeletal muscle capillarity and exercise capacity. Exp Physiol 94, 749-760.

Meek TH, Lonquich BP, Hannon RM \& Garland T Jr (2009). Endurance capacity of mice selectively bred for high voluntary wheel running. J Exp Biol 212, 2908-2917.
Olfert IM \& Birot O (2011). Importance of anti-angiogenic factors in the regulation of skeletal muscle angiogenesis. Microcirculation 18, 316-330.

Olfert IM, Breen EC, Gavin TP \& Wagner PD (2006). Temporal thrombospondin-1 mRNA response in skeletal muscle exposed to acute and chronic exercise. Growth Factors 24, 253-259.

Olfert IM, Breen EC, Mathieu-Costello O \& Wagner PD (2001). Skeletal muscle capillarity and angiogenic mRNA levels after exercise training in normoxia and chronic hypoxia. J Appl Physiol 91, 1176-1184.

Olfert IM, Howlett RA, Tang K, Dalton ND, Gu Y, Peterson KL, Wagner PD \& Breen EC (2009). Muscle-specific VEGF deficiency greatly reduces exercise endurance in mice. J Physiol 578, 1755-1767.

Olfert IM, Howlett RA, Wagner PD \& Breen EC (2010). Myocyte vascular endothelial growth factor is required for exercise-induced skeletal muscle angiogenesis. Am J Physiol Regul Integr Comp Physiol 299, R1059-R1067.

Prior SJ, Hagberg JM, Paton CM, Douglass LW, Brown MD, McLenithan JC \& Roth SM (2006). DNA sequence variation in the promoter region of the VEGF gene impacts VEGF gene expression and maximal oxygen consumption. Am J Physiol Heart Circ Physiol 290, H1848-H1855.

Rezende EL, Garland T Jr, Chappell MA, Malisch JL \& Gomes FR (2006a). Maximum aerobic performance in lines of Mus selected for high wheel-running activity: effects of selection, oxygen availability and the mini-muscle phenotype. J Exp Biol 209, 115-127.

Rezende EL, Gomes FR, Malisch JL, Chappell MA \& Garland T $\mathrm{Jr}(2006 b)$. Maximal oxygen consumption in relation to subordinate traits in lines of house mice selectively bred for high voluntary wheel running. J Appl Physiol 101, 477-485.

Richardson RS, Wagner H, Mudaliar SR, Henry R, Noyszewski EA \& Wagner PD (1999). Human VEGF gene expression in skeletal muscle: effect of acute normoxic and hypoxic exercise. Am J Physiol Heart Circ Physiol 277, H2247-H2252.

Rosenblatt JD, Kuzon WM, Plyley MJ, Pynn BR \& McKee NH (1987). A histochemical method for the simultaneous demonstration of capillaries and fiber type in skeletal muscle. Stain Technol 62, 85-92.

Swallow JG, Carter PA \& Garland T Jr (1998a). Artificial selection for increased wheel-running behavior in house mice. Behav Genet 28, 227-237.

Swallow JG, Garland T Jr, Carter PA, Zhan WZ \& Sieck GC (1998b). Effects of voluntary activity and genetic selection on aerobic capacity in house mice (Mus domesticus). J Appl Physiol 84, 69-76.

Swallow JG, Hayes JP, Koteja P \& Garland T (2009). Selection experiments and experimental evolution of performance and physiology. In Experimental Evolution: Concepts, Methods, and Application of Selection Experiments, ed. Garland TJ \& Rose MR, pp. 301-351. University of California Press, Berkeley, CA, USA.

Swallow JG, Koteja P, Carter PA \& Garland T (1999). Artificial selection for increased wheel-running activity in house mice results in decreased body mass at maturity. J Exp Biol 202, 2513-2520. 
Swallow JG, Koteja P, Carter PA \& Garland T Jr (2001). Food consumption and body composition in mice selected for high wheel-running activity. J Comp Physiol B 171, 651-659.

Syme DA, Evashuk K, Grintuch B, Rezende EL \& Garland T Jr (2005). Contractile abilities of normal and "mini" triceps surae muscles from mice (Mus domesticus) selectively bred for high voluntary wheel running. J Appl Physiol 99, 1308-1316.

Tang K, Breen EC, Gerber HP, Ferrara NM \& Wagner PD (2004). Capillary regression in vascular endothelial growth factor-deficient skeletal muscle. Physiol Genomics 18, 63-69.

Wagner PD (2003). Heterogeneity of skeletal muscle perfusion and metabolism. J Appl Physiol 95, 22022203.

Waters RE, Rotevatn S, Li P, Annex BH \& Yan Z (2004). Voluntary running induces fiber type-specific angiogenesis in mouse skeletal muscle. Am J Physiol Cell Physiol 287, C1342-C1348.
Wisloff U, Najjar SM, Ellingsen O, Haram PM, Swoap S, Al-Share Q, Fernstrom M, Rezaei K, Lee SJ, Koch LG \& Britton SL (2005). Cardiovascular risk factors emerge after artificial selection for low aerobic capacity. Science 307, 418-420.

Wong LE, Garland T Jr, Rowan SL \& Hepple RT (2009). Anatomic capillarization is elevated in the medial gastrocnemius muscle of mighty mini mice. J Appl Physiol 106, 1660-1667.

\section{Acknowledgements}

The authors wish to thank Harrieth Wagner and Darryl Neusome for their assistance in the laboratory in processing muscle tissue samples, and we thank Daniel Fulks for critically reading the manuscript. This project was supported by funding from West Virginia University School of Medicine (I.M.O.), TobaccoRelated Disease Research Program Grant 14KT-0091 (I.M.O.), American Heart Association Grant 10BGIA3630002 (I.M.O.) and National Science Foundation (IOB-0543429). 


\section{Viewpoint}

\section{Genetic background, endurance performance and muscle capillarization: lessons from the 'mini mice'}

\section{Olivier Birot}

\section{Email: birot@yorku.ca}

Genetic background contributes to endurance performance in humans. This has also been well established in rodent models by selecting lines of mice for their high exercise capacity. The 'mini mice' used in the study by Audet et al. (2011) in this issue of Experimental Physiology came from the 54th generation of mouse lines selected since the early 1990s for their exercise capacity in wheel-running cages (Swallow et al. 1998). These mice run 2.7-3 times further than the control lines and have an increased mitochondrial enzymatic activity, as well as increased maximal oxygen consumption, when tested in hypoxia. This selection process over the years has also led to an unexpected 'mini-muscle' phenotype due to a single Mendelian recessive allele and characterized by a $50 \%$ reduction of the hindlimb muscle mass and a lower body weight. When compared with control lines, certain hindlimb muscles, such as the gastrocnemius, have a decreased weight relative to body weight and a higher proportion of slow oxidative fibres (Audet et al. 2011).

Skeletal muscle angio-adaptation refers to the ability of capillaries to regress, stabilize or grow in order to match blood and oxygen supply with the metabolic needs of the myofibres. These processes are tightly regulated by a balance between pro- and anti-angiogenic factors, such as vascular endothelial growth factor-A (VEGF-A) and thrombospondin-1 (TSP-1), respectively.

By determining the oxygen conductance to myofibres, the capillary-to-fibre interface appears to be a crucial component of skeletal muscle function and endurance exercise performance. There are two ways to improve the capillary-to-fibre interface. As observed in response to endurance training, the growth of capillaries by the process of angiogenesis leads to an increase in the capillary-to-fibre ratio $(\mathrm{C} / \mathrm{F})$, thus enhancing the capillary-tofibre interface. Alternatively, in the case of fixed $\mathrm{C} / \mathrm{F}$ but decreased myofibre crosssectional area, as observed in the muscles of bats or humminbirds, capillary density will increase as a direct consequence of alteration of the myofibres, resulting in a higher capillary-to-fibre interface; in this situation, however, there is no de novo capillary formation (i.e. angiogenesis).

Since 'mini mice' have a significantly increased exercise capacity, Wong et al. (2009) have recently analysed the level of capillarization in the medial gastrocnemius muscle from these animals. Interestingly, the capillary-to-fibre interface was increased by region-specific mechanisms within the same muscle. In the superficial part of the medial gastrocnemius, a reduction in the myofibre size was responsible for an increased capillary density, whereas elsewhere angiogenesis around individual fibres increased the capillary-to-fibre ratio. Overall, muscle capillarization was improved and was suggested to contribute to the high exercise capacity of 'mini mice'.

As a natural follow-up step, Audet et al. (2011) hypothesized that the expression of VEGF-A and TSP-1, the key pro- and anti-angiogenic factors regulating skeletal muscle angio-adaptation, would be altered in 'mini mouse' skeletal muscles.

They first confirmed that gastrocnemius and plantaris muscles from 'mini mice' taken at rest indeed had higher $\mathrm{C} / \mathrm{F}$ and capillary density than control muscles. As expected, these muscles also expressed higher basal levels of VEGF-A compared with control animals. More interesting, however, was the VEGF-A expression level in response to one bout of intense running exercise. Exercise is a wellestablished stimulus for VEGF-A expression in human and rodent skeletal muscle. Here, the authors proposed two interesting hypotheses. First, VEGF-A levels were already higher in mini-muscles than in control muscles in resting conditions. In addition, because of their higher level of capillarization, mini-muscles would be less sensitive to exercise-induced hypoxic stress. As VEGF-A expression is highly regulated at the transcriptional level by hypoxia and hypoxia-inducible factor- $1 \alpha$, one would therefore expect a lack of VEGFA responsiveness to exercise stimulus in mini-muscles, and thus an increase in exercised control animals only. Second, mini-muscles have higher VEGF-A and capillarization levels at rest, but they also have higher oxidative activity compared with control muscles. Exercise would then stimulate VEGF-A expression in both control and mini-muscles to a similar relative extent. I will keep some suspense here and encourage the reader to find the answer in the article by Audet et al. (2011). Regarding the expression of the antiangiogenic TSP-1, the results are less clear and seem to be muscle-type specific. At rest, TSP-1 levels were similar in gastrocnemius muscles from control and 'mini mice'. In response to one bout of acute exercise, however, TSP-1 level was dramatically decreased in exercised mini-muscles. In the plantaris muscle, basal TSP-1 level was lower in 'mini mice' than in control mice, but exercise stimulated TSP-1 expression to a similar extent in both control and 'mini mice'.

Taken together, the results of Audet et al. (2011) illustrated that the angio-adaptive balance is altered in favour of some proangiogenic activity in mice selectively bred for high exercise capacity. This is a novel and interesting finding about the influence of the genetic background on muscle physiology and exercise performance.

This study also brings exciting questions. Why is basal VEGF-A expression higher in skeletal muscles from 'mini mice'? Could it be due to some genetic influences affecting VEGF-A gene directly? For example, Prior et al. (2006) have recently described that certain polymorphisms in VEGF-A DNA sequence were correlated with higher maximal oxygen consumption in human subjects. We could also not exclude the possibility that some genetic influences might indirectly affect VEGF-A expression. Prior et al. (2003) have shown DNA sequence variations for hypoxia-inducible factor- $1 \alpha$ that were also correlated with maximal oxygen consumption in human subjects. To what extent could these alterations affect hypoxia-inducible factor$1 \alpha$ function towards its targets, such as VEGF-A? 
There is no doubt that Audet et al. (2011) have open a bit wider the door of an exciting but unfortunately understudied aspect of muscle angio-adaptation to exercise.

\section{References}

Audet GN, Meek TH, Garland T Jr \& Olfert IM (2011). Expression of angiogenic regulators and skeletal muscle capillarity in selectively bred high aerobic capacity mice. Exp Physiol 96, 1138-1150.
Prior SJ, Hagberg JM, Paton CM, Douglass LW, Brown MD, McLenithan JC \& Roth SM (2006). DNA sequence variation in the promoter region of the $V E G F$ gene impacts VEGF gene expression and maximal oxygen consumption. Am J Physiol Heart Circ Physiol 290, H1848-H1855.

Prior SJ, Hagberg JM, Phares DA, Brown MD, Fairfull L, Ferrell RE \& Roth SM (2003). Sequence variation in hypoxia-inducible factor 1alpha (HIF1A): association with maximal oxygen consumption. Physiol Genomics 15, 20-26.
Swallow JG, Carter PA \& Garland T Jr (1998). Artificial selection for increased wheel-running behavior in house mice. Behav Genet 28, 227-237.

Wong LE, Garland T Jr, Rowan SL \& Hepple RT (2009). Anatomic capillarization is elevated in the medial gastrocnemius muscle of mini mice. J Appl Physiol 106, 1660-1667. 